\title{
Sobre Estructuras de Dirac en Espacios Vectoriales
}

\author{
José Crispín Ruíz Pantaleón \\ Universidad de Sonora \\ e-mail: jcpanta@mat.uson.mx
}

\begin{abstract}
Resumen
Se presenta la definición de una estructura de Dirac en un espacio vectorial y algunos ejemplos de esta clase de estructura geométrica. Se motiva la definición desde el contexto de la teoría de circuitos utilizando un circuito eléctrico concreto cuyas corrientes eléctricas y voltajes están sujetas a las denominadas leyes de Kirchhoff.
\end{abstract}

\section{Introducción}

El propósito de este escrito es presentar la definición de una estructura de Dirac en un espacio vectorial, o estructura de Dirac lineal, y algunos ejemplos de este tipo de estructuras. Con la intención de motivar la definición formal y resaltar la naturalidad y el alcance que poseen las estructuras de Dirac en el estudio de algunos sistemas (físicos), utilizamos un circuito eléctrico concreto en el que son aplicables las leyes de Kirchhoff para construir una estructura de Dirac que, como veremos, codifica todos los posibles valores para las corrientes eléctricas y los voltajes que admite el circuito y que están sometidas a las restricciones impuestas por estas leyes [8].

En analogía con la filosofía de optar por un estudio cualitativo de los sistemas de ecuaciones diferenciales (no lineales, en general), debido a la dificultad que presenta el resolverlos, el estudio de los sistemas físicos se ha inclinado por un enfoque geométrico. Esto es, realizar esfuerzos por comprender la geometría que subyace en los modelos de tales sistemas. Esto ha dado lugar a distintas clases de estructuras geométricas, tales como las estructuras simplécticas, estructuras de Poisson o las ya mencionadas estructuras de Dirac [9]. En general, éstas se definen en variedades diferenciales, modelo del espacio de estados de un sistema, y la geometría que originan son un tipo especial de foliaciones (de las variedades), a saber, foliaciones simplécticas y pre-simplécticas [7]. Sin embargo, en este texto sólo nos centraremos en presentar las estructuras de Dirac lineales, es decir, las definidas en espacios vectoriales. Que de hecho, resultan ser una clase especial de variedades.

El concepto de estructura de Dirac fue considerado inicialmente por Courant y Weinstein $[3,4]$. El objetivo original de estos autores fue encontrar una generalización común de las estructuras pre-simplécticas y Poisson. Adicionalmente, se introdujo la noción de estructura de Dirac para incluir restricciones en el estudio de sistemas en mecánica clásica. Por ejemplo, restricciones inducidas por Lagrangianos degenerados, investigación realizada por Dirac [5] 
y razón del nombre de estas estructuras. Otro ejemplo, son los circuitos eléctricos en los que se involucran tanto restricciones primarias como restricciones provenientes de las leyes de Kirchhoff. Posteriormente, Dorfman [6] introdujo un análogo algebraico de la noción de estructura de Dirac que fue empleado en la construcción de un formalismo algebraico de las estructuras Hamiltonianas que permite establecer un marco adecuado para el estudio de sistemas dinámicos con espacios fase de dimensión infinita que prescinde de la naturaleza de estos espacios.

En años recientes las estructuras de Dirac han ayudado al modelado y control de sistemas físicos (abiertos) y a la descripción en formalismo Hamiltoniano de sistemas con ligaduras. En particular, a la comprensión de los llamados sistemas Hamiltonianos con puertos, que están basados en la combinación de ideas y técnicas provenientes de la teoría de sistemas Hamiltonianos y de la teoría de redes, teniendo esta última su origen en la ingeniería eléctrica $[10,11,1,12,9,2]$. Enfatizamos que un punto importante en todo lo anterior es el considerar dentro del análisis del modelo de un sistema físico restricciones (relaciones) que pueden aparecer entre algunas de las variables del modelo.

\section{Espacio de Corrientes y Voltajes}

El objetivo de esta sección es presentar una manera de cómo la información sobre las corrientes eléctricas y los voltajes de un circuito eléctrico puede ser codificada en una estructura geométrica que denominamos espacio de corrientes y voltajes. Este espacio resulta ser un caso particular de una clase de estructuras geométricas más generales llamadas estructuras de Dirac, las cuales surgen de manera natural en el estudio de sistemas (físicos) con restricciones, es decir, sistemas en los que se presentan ciertas relaciones entre algunas variables que los modelan. Para el caso de los circuitos eléctricos, las leyes de Kirchhoff dan lugar a ciertas restricciones que involucran a las corrientes y los voltajes. Por esto, la primera parte de esta sección está dedicada a presentar un breve recordatorio de tales leyes y un ejemplo de cómo aplicarlas.

\subsection{Leyes de Kirchhoff}

Las denominadas leyes de Kirchhoff, aplicables al cálculo de corrientes, voltajes y resistencias de una malla eléctrica, establecen bases en la teoría de circuitos para un estudio de estos independiente de los elementos contenidos en las ramas. Esto sitúa a la teoría de grafos como una importante herramienta para el análisis (combinatorio) de los circuitos eléctricos. Bajo este contexto, un circuito se puede pensar como un grafo donde los vértices y las aristas se corresponden naturalmente con los nodos y las ramas del circuito, respectivamente. Aún más, una vez asignado un sentido positivo para la corriente eléctrica lo que se obtiene es un grafo dirigido.

Recordemos que la primera ley de Kirchhoff, o ley de corrientes eléctricas, afirma que en cualquier nodo de un circuito eléctrico la suma algebraica de las corrientes que pasan por el 
nodo es nula. En otras palabras, la suma de las corrientes que "entran" a un nodo es igual a la suma de las corrientes que "salen" del mismo. En la Figura 1 se presenta un esquema de esta afirmación.

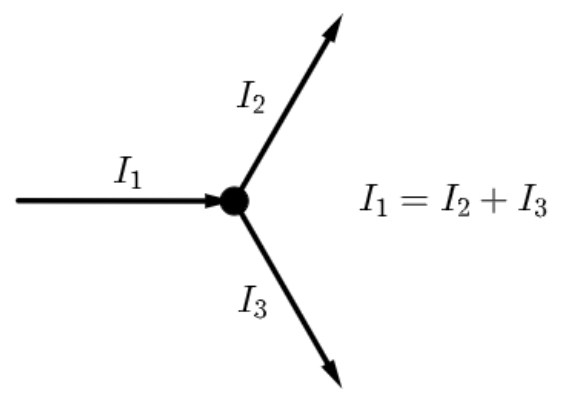

Figura 1: Primera Ley de Kirchhoff

Por su parte, la segunda ley de Kirchhoff, o ley de voltajes, establece que la suma algebraica de las caídas de voltaje a lo largo de un circuito cerrado es nula. De manera equivalente, que el voltaje $(v)$ en una rama del circuito es igual a la diferencia de los potenciales eléctricos $\left(u_{k}\right)$ en los nodos $\left(n_{k}\right)$ que conecta tal rama, en este caso, definido en el sentido de la corriente eléctrica, $k=1,2$. En la Figura 2 se presenta un esquema de esta afirmación.

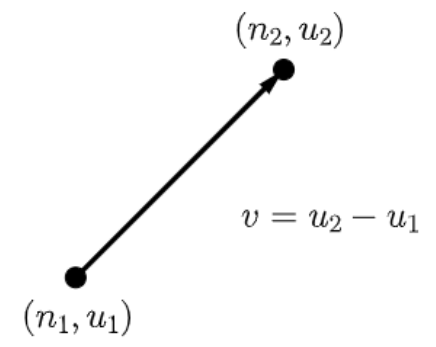

Figura 2: Segunda Ley de Kirchhoff

Observaciones 2.1 Aquí, se dirá que una arista dirigida como en la Figura 2 "comienza" en el vértice $n_{1} y$ "termina" en el vértice $n_{2}$.

Como ejemplo consideremos el siguiente grafo dirigido, 


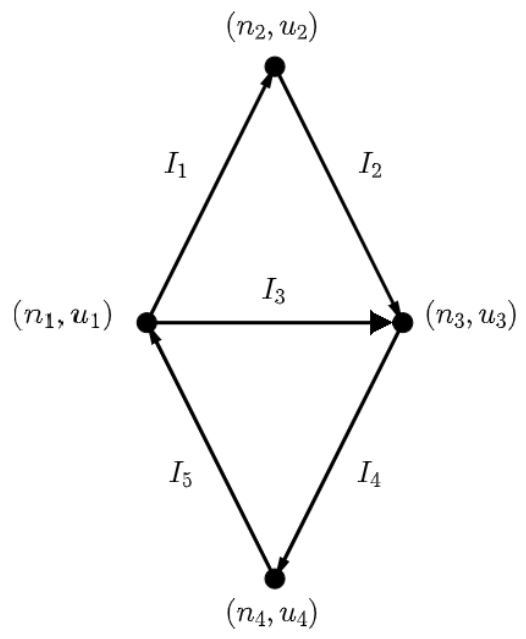

Figura 3: Grafo dirigido de un circuito eléctrico.

donde a cada vértice $n_{k}$ se le ha asignado un potencial eléctrico $u_{k}$ y a cada arista $l$ una corriente eléctrica $I_{l}$, con $k=1, \ldots, 4 ; l=1, \ldots, 5$. Así, de la primera ley de Kirchhoff se obtiene el sistema de ecuaciones (lineales)

$$
\left\{\begin{aligned}
-I_{1}-I_{3}+I_{5} & =0 \\
I_{1}-I_{2} & =0 \\
I_{2}+I_{3}-I_{4} & =0 \\
I_{4}-I_{5} & =0
\end{aligned}\right.
$$

y de la segunda ley de Kirchhoff el sistema (lineal)

$$
\left\{\begin{array}{l}
u_{2}-u_{1}=v_{1}, \\
u_{3}-u_{2}=v_{2}, \\
u_{3}-u_{1}=v_{3}, \\
u_{4}-u_{3}=v_{4}, \\
u_{1}-u_{4}=v_{5},
\end{array}\right.
$$

donde cada $v_{l}$ denota el voltaje asignado a la arista $l$.

Formulación Matricial. A continuación, se presenta una manera de expresar las leyes de Kirchhoff en términos de las llamadas matrices de incidencia, asociadas a grafos dirigidos, las cuales permiten capturar la estructura combinatoria de los circuitos eléctricos.

Si bien los sistemas de ecuaciones lineales (1) y (2) admiten una representación matricial inmediata, en este apartado se ha optado por presentar tal representación desde el punto de vista de la teoría de gráficas con la intención de ilustrar (de manera elemental) cómo esta teoría puede servir como herramienta para el estudio de ciertos sistemas. Concretamente, los sistemas de ecuaciones (1) y (2) se pueden expresar, respectivamente, por

$$
A I=0 \quad \text { y } \quad A^{\top} u=v
$$


donde se han definido $I:=\left(I_{1}, \ldots, I_{5}\right)^{\top}$ el vector de corrientes, $u:=\left(u_{1}, \ldots, u_{4}\right)^{\top}$ el (co)vector de potenciales, $v:=\left(v_{1}, \ldots, v_{5}\right)^{\top}$ el (co)vector de voltajes y la matriz $A=\left(a_{k l}\right)_{4 \times 5}$ por

$$
a_{k l}:=\left\{\begin{aligned}
-1 & \text { si la arista dirigida } l \text { "comienza" en el vértice } k, \\
1 & \text { si la arista dirigida } l \text { "termina" en el vértice } k, \\
0 & \text { en otro caso. }
\end{aligned}\right.
$$

Por definición, en este caso se tiene que

$$
A=\left(\begin{array}{rrrrr}
-1 & 0 & -1 & 0 & 1 \\
1 & -1 & 0 & 0 & 0 \\
0 & 1 & 1 & -1 & 0 \\
0 & 0 & 0 & 1 & -1
\end{array}\right)
$$

Observaciones 2.2 En teoría de grafos a la matriz A definida en (4) se le llama matriz de incidencia del (correspondiente) grafo dirigido. En este caso, no es difícil verificar que $\operatorname{rank} A=3$. En general, para grafos conexos, el rango de una matriz de incidencia es igual a \#renglones - 1 .

Así, en términos de la matriz de incidencia $A$ asociada al grafo dirigido (circuito eléctrico) de la Figura 3, las leyes de Kirchhoff se pueden reformular de manera equivalente por

$$
\begin{aligned}
1^{\text {ra }} \text { ley de Kirchhoff } & \Longleftrightarrow I \in \operatorname{ker} A, \\
2^{\text {da }} \text { ley de Kirchhoff } & \Longleftrightarrow v \in \operatorname{Im} A^{\top} .
\end{aligned}
$$

\subsection{Espacio de Corrientes y Voltajes}

En este apartado se presenta la definición de lo que denominamos espacio de corrientes y voltajes asociado a un circuito eléctrico, algunas de sus propiedades y finalmente una caracterización geométrica de este. Cabe mencionar que tal espacio captura todos los posibles valores para las corrientes eléctricas y los voltajes que admite un circuito eléctrico en los que son válidas las leyes de Kirchhoff. Entre las ventajas de la caracterización geométrica que se presenta del espacio de corrientes y voltajes se encuentra el poder deducir de manera sencilla importantes teoremas de la teoría de circuitos tales como el Teorema de Tellegen.

Primero, recordemos que, en general, cada matriz real induce de manera canónica una transformación lineal dada por multiplicación a la izquierda. Sea

$$
T_{A}: \mathbb{R}^{5} \longrightarrow \mathbb{R}^{4}, \quad x \longmapsto A x,
$$

la transformación lineal inducida por la matriz de incidencia $A$ asociada al grafo de la Figura 3 y sea

$$
T_{A}^{*}: \mathbb{R}^{4^{*}} \longrightarrow \mathbb{R}^{5^{*}},
$$


la transformación dual de $T_{A}$, donde $\mathbb{R}^{s *}$ denota el espacio dual de $\mathbb{R}^{s}, s=4,5$. Como es conocido se cumple que $T_{A}^{*}=T_{A^{\top}}$.

Bajo estos términos, se sigue de manera inmediata de (5) y (6) que el conjunto de todas las (posibles) corrientes y voltajes permitidas por las leyes de Kirchhoff para el grafo de la Figura 3 es descrito por el conjunto

$$
D^{A}:=\left\{(I, v) \in \mathbb{R}^{5} \times \mathbb{R}^{5^{*}} \mid I \in \operatorname{ker} T_{A}, v \in \operatorname{Im} T_{A}^{*}\right\} \subset \mathbb{R}^{5} \times \mathbb{R}^{5^{*}}
$$

el cual resulta ser un subespacio vectorial de $\mathbb{R}^{5} \times \mathbb{R}^{5^{*}}$ con las operaciones (inducidas) de suma y multiplicación por escalar usuales. Por su relevancia, a este subespacio lo denominaremos espacio de corrientes y voltajes asociado al grafo de la Figura 3.

Notemos que

$$
D^{A}=\operatorname{ker} T_{A} \times\left(\operatorname{ker} T_{A}\right)^{\circ},
$$

donde $\left(\operatorname{ker} T_{A}\right)^{\circ}=\left\{\alpha \in \mathbb{R}^{5^{*}} \mid \alpha(x)=0, \forall x \in \operatorname{ker} T_{A}\right\}$ denota el subespacio anulador del kernel de $T_{A}$. En particular, se tiene que

$$
\operatorname{dim} D^{A}=5=\operatorname{dim} \mathbb{R}^{5} .
$$

En efecto, tomando en cuenta que $\left(\operatorname{ker} T_{A}\right)^{\circ}=\operatorname{Im} T_{A}^{*}$, por la definición de $D^{A}$ en (7), se tiene que $D^{A}=\operatorname{ker} T_{A} \times \operatorname{Im} T_{A}^{*}=\operatorname{ker} T_{A} \times\left(\operatorname{ker} T_{A}\right)^{\circ}$. En otras palabras, la igualdad en (8) dice que todos los posibles valores para las corrientes y los voltajes en las aristas del grafo de la Figura 3 quedan codificados en subespacios vectoriales ortogonales (respecto al producto interno usual en $\mathbb{R}^{5}$ ).

Una consecuencia inmediata de lo anterior es el conocido Teorema de Tellegen, el cual se reformula en este caso diciendo que

$$
v^{\top} I=0,
$$

para todo $(I, v) \in D^{A}$. Este teorema, recordemos, expresa que la energía en el circuito no se crea ni se destruye por el hecho de conectar los nodos a través de las ramas del mismo, sin contradecir el que las ramas puedan contener resistencias o fuentes.

Observaciones 2.3 Como en (7) y (10), en esta sección se identificará $\mathbb{R}^{5^{*}} \simeq \mathbb{R}^{5}$ según convenga.

Geometría de $D^{A}$. En este apartado se muestra que las propiedades (9) y (10) permiten dar una caracterización geométrica del espacio de corrientes y voltajes definido en (7), propósito central de la presente sección. 
Primero, recordemos que dada una forma bilineal simétrica $B: E \times E \rightarrow \mathbb{R}$ en un espacio vectorial real $E$, se define el complemento ortogonal de un subespacio $W \subseteq E$ respecto a $B$ por

$$
W^{\perp}:=\{e \in E \mid B(e, w)=0, \forall w \in W\}
$$

Proposición 2.1 El subespacio de corrientes y voltajes $D^{A}$ definido en (7) satisface que

$$
\left(D^{A}\right)^{\perp}=D^{A}
$$

respecto a la forma bilineal (simétrica) $《, \Downarrow$ en $\mathbb{R}^{5} \times \mathbb{R}^{5^{*}}$ definida por $\left.《(x, \alpha),(y, \beta)\right\rangle:=$ $\alpha^{\top} y+\beta^{\top} x$.

Prueba. Primero, sea $(I, v) \in D^{A}$. Luego, para cualquier $(\widetilde{I}, \widetilde{v}) \in D^{A}$, se sigue de $(10)$ que $\langle(I, v),(\widetilde{I}, \widetilde{v})\rangle=v^{\top} \widetilde{I}+\widetilde{v}^{\top} I=0$. Lo que prueba que $D^{A} \subseteq\left(D^{A}\right)^{\perp}$. La igualdad en esta contención se sigue de $(9)$.

Si bien el Teorema de Tellegen ya advierte propiedades de ortogonalidad para los elementos del subespacio $D^{A}$, la proposición precedente caracteriza a este subespacio mediante esta propiedad respecto a una forma bilineal inducida por el producto interno usual en $\mathbb{R}^{5}$. Permitiendo lo anterior un estudio geométrico de los circuitos eléctricos en los que valen las leyes de Kirchhoff.

Observaciones 2.4 Claramente el número de vértices y aristas no juega un papel vital en la definición del subespacio $D^{A}$ en (7) ni en la derivación de sus propiedades. Sin embargo, se ha decidido trabajar a lo largo de esta sección con la configuración de la Figura 3 con el propósito de ilustrar lo mejor posible las construcciones realizadas. La generalización a un número arbitrario de vértices y aristas es análoga.

\section{$3 \quad$ Estructuras de Dirac en Espacios Vectoriales}

En la sección precedente se mostró cómo en el estudio topológico de los circuitos eléctricos, en los que valen las leyes de Kirchhoff, surge de manera natural un tipo especial de estructura geométrica caracterizada por una condición de ortogonalidad (Proposición 2.1), la cual codifica las restricciones en corrientes eléctricas y voltajes impuestas por las leyes de Kirchhoff. Esta situación no es única, estructuras geométricas análogas surgen también en el estudio de diversos sistemas físicos que presentan ciertas restricciones entre las variables que los modelan. Por ejemplo, sistemas mecánicos con restricciones holonómicas. Lo anterior sugiere encontrar un marco adecuado que formalice esta clase de estructuras geométricas. Esto da lugar a las llamadas estructuras de Dirac en espacios vectoriales cuya definición es el punto central de esta sección. 
Sean $V$ un espacio vectorial real finito dimensional y $V^{*}$ su espacio dual. Definamos en el espacio vectorial $V \times V^{*}$ la forma bilineal simétrica

$$
\langle,\rangle:\left(V \times V^{*}\right) \times\left(V \times V^{*}\right) \longrightarrow \mathbb{R}, \quad\langle(x, \alpha),(y, \beta)\rangle:=\langle\alpha, y\rangle+\langle\beta, x\rangle,
$$

inducida por el pairing natural $\langle\eta, z\rangle:=\eta(z)$ entre vectores $z \in V$ y funcionales lineales $\eta \in V^{*}$. Notemos la similitud entre esta forma bilineal y la definida en la Proposición 2.1.

Es sencillo verificar que la forma bilineal (12) es no-degenerada pero, en general, no es positiva ni negativa definida. En efecto, si $(x, \alpha)$ es tal que $\langle(x, \alpha),(y, \beta)\rangle=0$, para todo $(y, \beta) \in V \times V^{*}$; se sigue, en particular, que $\langle(x, \alpha),(y, 0)\rangle=\langle\alpha, y\rangle=0$, para todo $y \in V$. Esto implica que $\alpha=0$. Análogamente, se muestra que debe ser $x=0$. En conclusión, es $(x, \alpha)=0$. Lo que prueba la no degeneración de la forma bilineal (12). Ahora, dado que $\langle(x, \alpha),(x, \alpha)\rangle=2\langle\alpha, x\rangle$ admite cualquier signo, la forma bilineal (12) no es positiva ni negativa definida, en general.

Definición 3.1 Una estructura de Dirac en $V$ es un subespacio $D \subset V \times V^{*}$ tal que

$$
D^{\perp}=D
$$

donde $D^{\perp}$ denota el complemento ortogonal de $D$ respecto a la forma bilineal (12).

Notemos que bajo esta definición, por la Proposición 2.1, el espacio de corrientes y voltajes $D^{A}$ en (7) es una estructura de Dirac en $\mathbb{R}^{5}$. Aún más, resulta un caso particular de una clase especial de estructuras de Dirac las cuales se presentan en el siguiente ejemplo.

Ejemplo 3.2 Sea $V$ un espacio vectorial finito dimensional y sea $W \subseteq V$ un subespacio vectorial de $V$. Entonces,

$$
D:=W \times W^{\circ}
$$

es una estructura de Dirac en $V$, donde $W^{\circ} \subseteq V^{*}$ denota el (sub)espacio anulador de $W$. En efecto, por definición, es claro que $D \subseteq D^{\perp}$. Ahora, dado $(x, \alpha) \in D^{\perp}$, se tiene que $\langle(x, \alpha),(w, 0)\rangle=\langle\alpha, w\rangle=0$, para todo $w \in W$. En consecuencia, $\alpha \in W^{\circ}$. Análogamente, se tiene que $\langle(x, \alpha),(0, \omega)\rangle=\langle\omega, x\rangle=0$, para todo $\omega \in W^{\circ}$. Lo que implica que $x \in$ $\left(W^{\circ}\right)^{\circ}=W$. Por lo tanto, $(x, \alpha) \in D$. Lo que prueba que $D^{\perp} \subseteq D$. Mostrando así que se tiene la igualdad $D^{\perp}=D$.

Notemos que de (13) se sigue, en particular, que $V \times\{0\}$ y $\{0\} \times V^{*}$ son (siempre) estructuras de Dirac en $V$. 
Una consecuencia inmediata de la definición (13) es que

$$
\langle\alpha, x\rangle=0
$$

para todo $(x, \alpha) \in D$. En efecto, de (13) se sigue que $\langle(x, \alpha),(x, \alpha)\rangle=2\langle\alpha, x\rangle=0$. En el contexto de circuitos eléctricos esta propiedad no es más que el Teorema de Tellegen (10). Aún más, por la no degeneración de la forma bilineal (12), se sigue también que

$$
\operatorname{dim} D=\operatorname{dim} V
$$

tal como sucede en (9). Esto se deduce de la fórmula $\operatorname{dim} D+\operatorname{dim} D^{\perp}=\operatorname{dim} V \times V^{*}=$ $2 \operatorname{dim} V$. A continuación, se probará que las propiedades (14) y (15) caracterizan completamente a toda estructura de Dirac.

Proposición 3.3 Sean $V$ un espacio vectorial finito dimensional y $D \subset V \times V^{*}$ un subespacio vectorial. Las siguientes afirmaciones son equivalentes:

1. D es una estructura de Dirac en $V$.

2. $\operatorname{dim} D=\operatorname{dim} V \quad y \quad\langle\alpha, x\rangle=0$, para todo $(x, \alpha) \in D$.

Prueba. Es claro de (14) y (15) que el punto 1 implica 2. Ahora, supongamos válido el punto 2 y sean $(x, \alpha),(y, \beta) \in D$ arbitrarios. Notemos que $(x+y, \alpha+\beta) \in D$, por ser $D$ un subespacio vectorial. Luego, $\langle(x, \alpha),(y, \beta)\rangle=\langle\alpha, y\rangle+\langle\beta, x\rangle=\langle\alpha+\beta, x+y\rangle=0$. Esto implica que $D \subseteq D^{\perp}$. Finalmente, la igualdad $D=D^{\perp}$ se sigue de la hipótesis $\operatorname{dim} D=\operatorname{dim} V$. Probando así el punto 1.

Utilizando esta proposición resulta evidente que el subespacio en (13) es una estructura de Dirac. A continuación, utilizando la Proposición 3.3 se presentan algunos ejemplos adicionales de estructuras de Dirac.

Ejemplo 3.4 Consideremos el subespacio vectorial $D \subset \mathbb{R}^{2} \times \mathbb{R}^{2^{*}}$ dado por

$$
\begin{aligned}
D & :=\left\{\left((a, b)^{\top},(b,-a)\right) \mid(a, b)^{\top} \in \mathbb{R}^{2},(b,-a) \in \mathbb{R}^{2^{*}}\right\} \\
& \simeq \operatorname{span}\left\{(1,0,0,-1)^{\top},(0,1,1,0)^{\top}\right\} .
\end{aligned}
$$

Notemos que $\operatorname{dim} D=2=\operatorname{dim} \mathbb{R}^{2}$ y $(b,-a)(a, b)^{\top}=0$, para todo $\left((a, b)^{\top},(b,-a)\right) \in D$. Así, por la Proposición 3.3, es D una estructura de Dirac en $\mathbb{R}^{2}$. 
Ejemplo 3.5 Sea $T: V \rightarrow V^{*}$ un morfismo antisimétrico, esto es, tal que $T^{*}=-T$. Consideremos el subespacio $D^{T} \subset V \times V^{*}$ dado por la gráfica

$$
D^{T}:=\{(x, T x) \mid x \in V\} .
$$

Notemos que $\operatorname{dim} D^{T}=\operatorname{dim} V$ y $\langle T x, x\rangle=\left\langle T^{*} x, x\right\rangle=-\langle T x, x\rangle$, lo que implica que $\langle T x, x\rangle=$ 0 , para cualesquier $(x, T x) \in D^{T}$. Así, por la Proposición 3.3, es $D^{T}$ una estructura de Dirac en $V$.

Ejemplo 3.6 Sea $T: V \rightarrow V^{*}$ un morfismo antisimétrico. Dada una estructura de Dirac $D$ en $V$, sea

$$
\bar{D}:=\{(x, \alpha+T x) \mid(x, \alpha) \in D\} .
$$

Notemos que $\operatorname{dim} \bar{D}=\operatorname{dim} V y\langle\alpha+T x, x\rangle=\langle\alpha, x\rangle+\langle T x, x\rangle=0$, para cualesquier $(x, \alpha+$ $T x) \in \bar{D}$. Así, por la Proposición 3.3, es $\bar{D}$ una estructura de Dirac en $V$.

Ejemplo 3.7 Sea $E$ un espacio vectorial real finito dimensional y consideremos $V=E \times E^{*}$. Dado un subespacio vectorial $W \subset E$ definamos

$$
D:=\left\{((x, \alpha),(-\beta, x)) \mid x \in W, \alpha-\beta \in W^{\circ}\right\} \subset V \times V^{*} .
$$

Notemos que $\operatorname{dim} D=\operatorname{dim} V \quad y \quad\langle(-\beta, x),(x, \alpha)\rangle=\langle\alpha-\beta, x\rangle=0$, para cualesquier $((x, \alpha),(-\beta, x)) \in D$. Así, por la Proposición 3.3, es D una estructura de Dirac en $V$.

El siguiente ejemplo muestra una manera de inducir estructuras de Dirac en subespacios vectoriales de un espacio vectorial. La verificación del siguiente ejemplo se puede encontrar en [4].

Ejemplo 3.8 Dada una estructura de Dirac D en $V$ y un subespacio vectorial $W \subseteq V$, se puede inducir una estructura de Dirac $D_{W}$ en $W$ la cual resulta isomorfa al cociente

$$
D_{W} \simeq \frac{D \cap\left(W \oplus V^{\circ}\right)}{D \cap\left(\{0\} \oplus W^{\circ}\right)} .
$$

Concluimos esta sección mencionando que es posible dar una definición de una estructura de Dirac en una variedad diferencial (real) $M$. En esta definición, además de la condición de ortogonalidad (13), se pide una condición de compatibilidad con la estructura diferencial 
en $M$ (condición de integrabilidad). En este contexto, la geometría subyacente es la de una foliación presimpléctica de $M$. Esto generaliza las nociones de estructura simpléctica y estructura de Poisson, que son fundamentales en mecánica clásica, por ejemplo, y permite realizar un análisis de diversos sistemas físicos involucrando ciertas restricciones que los sistemas pueden presentar. Lo anteriormente expuesto por supuesto que invita a profundizar en el estudio de las estructuras de Dirac y sus posibles generalizaciones.

Agradecimientos. Un profundo agradecimiento al grupo de Geometría Diferencial y Sistemas Dinámicos de la Universidad de Sonora.

\section{Referencias}

[1] A. M. Bloch, P. S. Krishnaprasad, J. E. Marsden and R. Murray, Nonholonomic mechanical systems with symmetry, IFAC-PapersOnLine, 136, 21-99 (1996)

[2] A. M. Bloch, Nonholonomic Mechanics and Control, 484, Springer-Verlag, New York (2003)

[3] T. Courant and A. Weinstein, Beyond Poisson structures, Action hamiltoniennes de groupes. Troisième théorème de Lie, 27, 39-49 (1986)

[4] T. Courant, Dirac manifolds, Trans. Amer. Math. Soc., 319, 631-661 (1990)

[5] P. Dirac, Generalized Hamiltonian dynamics, Canadian J. Math., 2, 129-148 (1950)

[6] I. Dorfman, Dirac structures of integrable evolution equations, Physics Letters A., 125, 240-246 (1987)

[7] J. P. Dufour and N. T. Zung, Poisson Structures and their Normal Forms, 321, Birkhuser Basel (2005)

[8] D. Jeltsema and A. J. van der Schaft, Complexification of Dirac Structures and Generalised Kirchhoff Operators, IFAC-PapersOnLine, 48 - 13, 075-080 (2015)

[9] J. E. Marsden and T. S Ratiu, Introduction to Mechanics and Symmetry, 586, SpringerVerlag, New York (1999)

[10] A. J. van der Schaft and B. M. Maschke, On the Hamiltonian formulation of nonholonomic mechanical systems, Rep. on Math. Phys., 34, 225-233 (1994)

[11] A. J. van der Schaft and B. M. Maschke, The Hamiltonian formulation of energy conserving physical systems with external ports, Archiv für Elektronik und Ubertragungstechnik, 49, 362-371 (1995)

[12] A. J. van der Schaft, Implicit Hamiltonian systems with symmetry, Rep. Math. Phys., 41, 203-221 (1998) 